\title{
The ribosome-inactivating protein restrictocin deters insect feeding on Aspergillus restrictus
}

\author{
Tristan Brandhorst, ${ }^{1}$ Patrick F. Dowd ${ }^{2}$ and William R. Kenealy ${ }^{1}$ \\ Author for correspondence: William R. Kenealy. Tel: +1608223 0050. Fax: +16082313353.
}

$1 \mathrm{~J}$. Whittier Biologics, 2344 W Lawn Ave, Madison, WI 53711, USA

2 USDA-Agricultural Research Services, NCAUR, 1815 N University St, Peoria, IL 61604, USA
The fungus-feeding beetle, Carpophilus freemani, consumed equal quantities of young mycelia, fewer phialides bearing mature spores and much fewer phialides bearing developing spores of Aspergillus restrictus compared to those of Aspergillus nidulans when tested in diet choice assays. The degree to which specific fungal structures were consumed was inversely related to the localization of high levels of restrictocin, a ribosome-inactivating protein, to those structures. Pure restrictocin added to the insect diet at 1000 p.p.m. killed $38.5 \%$ of C. freemani larvae and $62.5 \%$ of Spodoptera frugiperda larvae in $\mathbf{4 8}$ h, but did not affect $C$. freemani adults or Helicoverpa zea larvae over the same interval. In diet choice assays, 1000 p.p.m. of restrictocin deterred feeding by adult $C$. freemani and Sitophilus zeamais compared to control diets. Thus, restrictocin production and localization may have a natural defensive role against insect feeding at times critical to spore formation by $A$. restrictus, and may have potential as an insect control agent.

Keywords: antifeedant, anti-insectan, pesticide, fungal defence

\section{INTRODUCTION}

Some species of fungi are more likely to be found associated with foodstuffs when certain insects are present. This has been shown to be the case with the sap beetles (Coleoptera: Nitidulidae) (Dowd, 1995), the granary weevil (Sitophilus granarius) (Agrawal et al., 1957), and mites (Astigmata: Acaridae) (Armitage \& George, 1986) on stored products. These insects inoculate clean grain with a variety of fungal spores present in their faeces and on the outsides of their bodies (Griffiths et al., 1959; Agrawal et al., 1957) and then feed upon the fungi that develop (Griffiths et al., 1959). Thus insects can simultaneously be important vectors for the spread of fungal infestation and fungal antagonists that restrict fungal growth through feeding.

Some fungi possess defences against insect predators that are analogous to the defences of higher plants (Wicklow, 1988). Secondary metabolites produced by the fungi can be toxic to insects, or deter insect feeding (Wright $e t$ al., 1982; Wicklow, 1988; Dowd, 1992a). While insects have adapted to feeding upon species of highly toxic fungi (Dowd 1992a, b), anti-insectan toxins can still contribute heavily to fungal defence (Wicklow et al., 1988). The sclerotia of Aspergillus flavus, for example, have been

Abbreviation: RIP, ribosome-inactivating protein. shown to contain substantial quantities of aflatoxin, as well as several aflavinine derivatives that deter insect predation (Wicklow et al., 1988).

Plant and fungal resistance to insects is often associated with low-molecular-mass chemicals. Proteins, however, are increasingly being found to contribute to insect resistance (Gatehouse et al., 1993). Ribosome-inactivating proteins (RIPs), such as ricin and saporin, are produced by a variety of higher plants (Stripe \& Barbieri, 1986). Both ricin and saporin were found to be toxic to selected beetle larvae, but not to caterpillar larvae (Gatehouse $e t$ al., 1990). It has also been suggested that the loss of RIPs in opaque-2 lines of corn has caused increased susceptibility to insect feeding (Bass et al., 1992).

Fungi also produce RIPs (Conde et al., 1978; Jimenez \& Vazquez, 1985) such as restrictocin, which is produced by Aspergillus restrictus. This fungus is often closely associated with beetle infestations of stored grain products (Agrawal et al., 1957). The production of restrictocin by A. restrictus is localized to the conidiophores and phialides during conidium formation (Brandhorst \& Kenealy, 1992). It has been suggested that the protein toxin localization could deter insects from feeding until conidia are fully formed, after which time fungus-feeding insects could externally or internally carry spores to new resources for the fungus (Brandhorst \& Kenealy, 1992).

In this study we examined the feeding of Carpopbilus 
freemani (an insect that preferentially feeds upon fungi; Tate \& Ogawa, 1975) upon Aspergillus nidulans and $A$. restrictus cultures of different developmental stages. We also examined the effect of pure restrictocin upon the health and feeding habits of various insects that feed on agricultural crops.

\section{METHODS}

Insects. Helicoverpa zea (corn earworms) and Spodoptera frugiperda (fall armyworms) were reared on pinto bean diet (Dowd, 1988); Carpophilus freemani were reared on pinto bean diet (Dowd \& Weber, 1991); Sitophilus zeamais (maize weevils) were reared on cracked corn (Dowd, 1994); all as previously described. Rearing conditions were $27 \pm 1{ }^{\circ} \mathrm{C}$, with a 14:10 light: dark cycle, at $50 \pm 10 \%$ relative humidity for the Sit. zeamais and $40 \pm 10 \%$ relative humidity for the other insect species.

Chemicals. The momordin, saporin, and ultra-low-temperaturegelling agarose were obtained from Sigma. The sorbic acid was obtained from US Biochemicals. The restrictocin was obtained as previously described (Brandhorst et al., 1994). All other chemicals were of reagent grade.

Fungal cultures. Aspergillus restrictus (strain NRRL 2869) and $A$. nidulans (strain WI: $\arg B, \operatorname{metH2}, b i A 1$ ) (Cullen $e t$ al., $1987 \mathrm{a}, \mathrm{b})$ were maintained and grown as previously described (Brandhorst et al., 1994). Agar plate fungal cultures were held at $22{ }^{\circ} \mathrm{C}$ for $8 \mathrm{~h}$ during week days, and at $4{ }^{\circ} \mathrm{C}$ the rest of the time in order to carefully observe development so that assays could be set up with the fungal cultures of the desired maturity (and thus restrictocin content). Fungi were grown both directly upon the agar surfaces of the culture plates and upon sterile filters (MSI, nitrocellulose, $0.4 \mu \mathrm{m}$ pore size) overlaying the agar.

Choice assays. Cultures used in choice assays with $C$. freemani adults included those in which all growth was mycelial, those in which conidiophores were in the early stages of conidiation, and those in which conidia were profuse and fully mature. Choice assays with adult $C$. freemani were set up in arenas consisting of $3.5 \mathrm{~cm}$ diameter Petri dishes. Squares (colonized by fungi) measuring $1 \times 1 \mathrm{~cm}$ were cut from the agar and placed on opposite sides of the plates. In the case of fungal material that had been grown on filter paper, $1 \times 1 \mathrm{~cm}$ squares were cut out and placed upon a $3 \mathrm{~cm}$ diameter piece of moistened filter paper in the bottom of the Petri dish. Five 1-week-old $C$. freemani adults were placed in the dishes and allowed to feed for $24 \mathrm{~h}$ on the fungal material covering the squares. Eight arenas of each treatment were used in each series of assays and each series was replicated for verification. Consumption was rated on an integer scale of 0 to 10 , with 0 corresponding to no detectable feeding, and 10 to all fungal material consumed. At the end of the $24 \mathrm{~h}$ period, the remaining fungal material was frozen for $24 \mathrm{~h}$ and then fixed in ethanol. The fixed material was examined microscopically to identify the remaining fungal structures.

Estimation of restrictocin content of differentiated structures. The mean number of conidiophores present on one agar plate culture of $A$. restrictus was estimated by microscopic examination of intact culture surfaces and homogenates of plategrown cell material. The mean number of phialides per conidiophore head was likewise estimated by microscopic examination. The mean length and diameter of the phialides, taken from both literature values (Frey et al., 1979) and microscopic measurements by the authors, were used to calculate the approximate volume and weight of the average phialide (assuming a cylindrical shape). The concentration of restrictocin upon the phialides could be estimated given the quantity of restrictocin present on agar plate cultures of $A$. restrictus (Brandhorst \& Kenealy, 1992).

Feeding activity and mortality assays. Pure restrictocin, momordin and saporin were incorporated in cold-gelled powdered corn at 1000 p.p.m. by a method described previously (Dowd, 1994). Briefly, the incorporation involved adding an acetone solution of sorbic acid (as a mould inhibitor) to the powdered corn (the concentration in the final product would be 1000 p.p.m.), evaporating the acetone, adding 2 parts water, and rehydrating at $4{ }^{\circ} \mathrm{C}$ overnight. A RIP was added in an additional part of water and the mixture was chilled at $4{ }^{\circ} \mathrm{C}$ for $2 \mathrm{~h}$. Ultralow-temperature-gelling agarose was added to a concentration of $5 \%(\mathrm{w} / \mathrm{v})$ and allowed to gel for at least $1 \mathrm{~h}$ before setting up in assays. For assays with adult $C$. freemani, approximately $0.6 \mathrm{~g}$ of material was used and five adult insects were included in each treatment. A feeding rating based on a 1-4 scale (Dowd, 1990) was used in feeding activity assays. For assays with Spo. frugiperda, 1.25 g of material was placed in dishes along with 20 newly hatched larvae per treatment. Larvae were examined for mortality after $48 \mathrm{~h}$. Assays with C. freemani larvae were run in a similar fashion to those for $S p$ o. frugiperda, except that secondinstar (approx. $0.5 \mathrm{mg}$ ) larvae were used. For assays with $H$. zea, 20 newly hatched larvae per treatment were individually caged in the wells of a 24-well tissue-culture plate with approximately $0.1 \mathrm{~g}$ of material. Plates were sealed as described previously (Dowd, 1988).

Feeding puncture assays. For assays with Sit. zeamais adults, cold-gelled corn powder was prepared as just described. Initial studies in choice-assays as described for $C$. freemani adults indicated relatively limited feeding over a 1 week period, and difficulty in counting the numbers of feeding punctures due to the friable nature of the material. A different diet was used that was of a smoother consistency so that feeding punctures could be counted. This diet was analogous to the pinto bean diet except that $240 \mathrm{~g}$ frozen sweet corn was substituted for the frozen pinto beans. A $5 \mathrm{~g}$ quantity of the diet was freeze dried (to $1.25 \mathrm{~g}$ dry weight) and then rehydrated with $3.75 \mathrm{ml}$ deionized water containing $5 \mathrm{mg}$ restrictocin (final concentration 1000 p.p.m.). An analogous control diet without restrictocin was also prepared. Each block was sectioned into eight pieces, and pairs of these pieces were set up in an arena test with 10 adult Sit. zeamais. The number of feeding punctures in each block was counted after 2 and $4 \mathrm{~d}$.

Digestion assays. The susceptibility of restrictocin to digestion by $H$. zea and Spo. frugiperda was determined by comparing degradation over a discrete time interval with different dilutions of gut homogenates. Midguts from three different last-instar caterpillars of each species were dissected individually in icecold $0.1 \mathrm{M}$ glycine buffer, $\mathrm{pH} 9 \cdot 6$. Each individual midgut was homogenized in $1 \mathrm{ml}$ of the same buffer using a ground-glass tissue grinder. The homogenate was centrifuged at $10000 \mathrm{~g}$ for $10 \mathrm{~min}$ at $4{ }^{\circ} \mathrm{C}$, and the supernatant (excluding any floating lipid layer) was used as an enzyme source. Protein determinations were done with the Bio-Rad packaged assay using bovine serum albumin as a standard. Protein content of homogenates used in assays was adjusted to $1 \mathrm{mg} \mathrm{ml}^{-1}( \pm 10 \%)$ by dilution with buffer. Incubations consisted of $25 \mu \mathrm{l}$ diluted homogenate and $25 \mu \mathrm{l} 1 \mathrm{mg} \mathrm{m}^{-1}$ solution of restrictocin in buffer. Enzyme-free restrictocin was also tested over the interval. All solutions were incubated at $27 \pm 1{ }^{\circ} \mathrm{C}$ for $1 \mathrm{~h}$, then frozen at $-20^{\circ} \mathrm{C}$ until examined electrophoretically.

Native polyacrylamide gel electrophoresis was performed as described previously (Dowd, 1994) with minor modifications. Because of the basic nature and small size of restrictocin, the gel was positioned with the sample slots nearest the anode, and 
electrophoresis was carried out for only $30 \mathrm{~min}$. The gel was removed, fixed in trichloroacetic acid/sulfosalicylic acid for $2 \mathrm{~h}$, rinsed rapidly three times with deionized water, stained overnight with Pharmacia PhastGel Blue R (Coomassie R350) (prepared according to directions included with the material) and destained with repeated changes of $30 \%(\mathrm{v} / \mathrm{v})$ methanol.

\section{RESULTS}

\section{Choice assays}

The presentation of insects with fungal cultures at specific developmental stages required careful observation of the cultures. This necessitated the overnight storage of plate cultures when there were no observers present. The cold storage of the plates did not dramatically alter the morphology of the fungal cultures and development proceeded according to the time of incubation at growth temperatures.

The C. freemani adults consumed the mycelia of both $A$. nidulans and $A$. restrictus with no noticeable discrimination (Table 1). Feeding by $C$. freemani adults on $A$. restrictus decreased somewhat when conidiophores were being formed (data not shown). When cultures entered the early stages of conidium formation, $C$. freemani adults fed extensively upon the phialides and the immature conidia of $A$. nidulans, as indicated by the marked absence of these structures after feeding. In comparison, these structures remained almost entirely unconsumed in cultures of $A$. restrictus. Overall, there was a marked decrease in feeding on $A$. restrictus compared to $A$. nidulans when conidia were starting to develop (Table 1). Although not shown, this was independent of the type of assay (filter paper vs agar), results not being significantly different. Shortly after the maturation of the conidia of $A$. restrictus, the adult $C$. freemani resumed feeding upon the phialides and fed upon the mature conidia as well; however, this level of feeding was still significantly lower than for $A$. nidulans (Table 1).

Table 1. Effects of fungal development on the feeding of $C$. freemani adults on $A$. nidulans and $A$. restrictus cultures

The feeding scores are based on a 0-10 scale, with 0 indicating no feeding. Values are means ( \pm standard errors) based on eight arenas used in each series of evaluations. Values for $A$. restrictus followed by an asterisk are significantly different from those of A. nidulans $(P<0.05)$ by paired $t$-test analysis.

\begin{tabular}{|lcc|}
\hline Growth stage of culture & \multicolumn{2}{c|}{ Feeding rating } \\
\cline { 2 - 3 } & A. nidulans & A. restrictus \\
\hline Mycelia & $10 \cdot 0 \pm 0 \cdot 0$ & $10 \cdot 0 \pm 0 \cdot 0$ \\
Immature conidia (on agar) & $9 \cdot 9 \pm 0 \cdot 1$ & $0 \cdot 9 \pm 0 \cdot 0^{*}$ \\
Immature conidia & $9 \cdot 5 \pm 0 \cdot 3$ & $0 \cdot 8 \pm 0 \cdot 2^{*}$ \\
(on filter overlay) & $9 \cdot 9 \pm 0 \cdot 1$ & $6 \cdot 0 \pm 0 \cdot 1^{*}$ \\
Mature conidia & \\
\hline
\end{tabular}

Table 2. Effects of restrictocin on feeding activity of $C$. freemani adults

Feeding activities are based on a 1-4 scale (Dowd, 1990) using cold gel incorporation. A score of 1 would indicate no feeding. Eight arenas were used in each series of evaluations. The results are means \pm standard errors.

\begin{tabular}{|cc|}
\hline $\begin{array}{c}\text { Restrictocin in diet } \\
\text { (p.p.m.) }\end{array}$ & Feeding activity \\
\hline 0 & $3 \cdot 2 \pm 0 \cdot 3$ \\
500 & $3 \cdot 1 \pm 0 \cdot 3$ \\
1000 & $2 \cdot 5 \pm 0 \cdot 4$ \\
2500 & $1 \cdot 6 \pm 0 \cdot 3$ \\
\hline
\end{tabular}

When presented with a choice of a diet containing restrictocin or a diet without additives, $C$. freemani adults were not significantly deterred from feeding on the restrictocin-containing diet until higher concentrations of restrictocin (1000 and 2500 p.p.m.) were used (Table 2).

\section{Estimation of restrictocin content of differentiated structures}

The concentration of restrictocin present upon the phialides of the conidiophores was estimated in order to compare the concentrations of restrictocin used in the artificial diets of the choice and mortality assays to the concentration present in $A$. restrictus. Cell-associated restrictocin reaches a peak concentration of $18 \mu \mathrm{g}$ per agar plate culture (Brandhorst \& Kenealy, 1992). At this time, there are an average of $1 \times 10^{7}$ conidiophores per plate and approximately 60 phialides per conidiophore. The volume of a phialide was estimated to be $21 \mu \mathrm{m}^{3}$. The product of these last three figures yields $12.6 \mathrm{~mm}^{3}$ of phialides per agar plate culture. If we assume that all the restrictocin is secreted by the phialides, the concentration of restrictocin upon the phialides may be approximated: $18 \mu \mathrm{g}$ restrictocin $/ 12.6 \mathrm{~mm}^{3}$ phialides $=1400$ p.p.m.

\section{Mortality assays}

Restrictocin (1000 p.p.m.) was used to test mortality of adult and larval forms of $C$. freemani. The mortality rates for $C$. freemani adults and $H$. zea larvae were not significantly affected by restrictocin at 1000 p.p.m. (Table 3 ). The mortality of Spo. frugiperda and C. freemani larvae feeding on the restrictocin-containing material was significant at $48 \mathrm{~h}$. No mortality of Spo. frugiperda larvae fed on gelled corn powder containing the RIPs momordin or saporin was noted after $48 \mathrm{~h}$ (Table 3 ).

\section{Feeding puncture assays}

Restrictocin at 1000 p.p.m. significantly reduced feeding by Sit. zeamais adults after $2 \mathrm{~d}$ and $4 \mathrm{~d}$. The number of feeding punctures in the restrictocin-containing diet was 
Table 3. Effects of different RIPs at 1000 p.p.m. on the mortality of different insect species

Values are based on both live and dead insects found. Values followed by an asterisk are significantly different from the control $(P<0.05)$ by chi-square analysis. $(N)$ indicates the number of insects included in each experiment.

\begin{tabular}{|llllll|}
\hline RIP & \multicolumn{1}{c}{ Insect species } & \multicolumn{3}{c|}{ Percentage mortality (48 h) } \\
\cline { 3 - 6 } & & Control & (N) & + RIP & (N) \\
\hline \multirow{2}{*}{ Restrictocin } & C. freemani adult & 0 & $(20)$ & 0 & $(20)$ \\
& C. freemani larvae & 0 & $(16)$ & $38 \cdot 5^{*}$ & $(13)$ \\
& H. zea larvae & 0 & $(20)$ & $5 \cdot 2$ & $(19)$ \\
& Spo. frugiperda larvae & 0 & $(14)$ & $62.5^{*}$ & $(16)$ \\
Momordin & Spo. frugiperda larvae & 4.8 & $(21)$ & 0 & $(19)$ \\
Saporin & Spo. frugiperda larvae & 4.8 & $(21)$ & 0 & $(18)$ \\
\hline
\end{tabular}

Table 4. Effects of restrictocin on feeding by adult Sit. zeamais

Values are means ( \pm standard errors) based on eight arenas used in each series of evaluations. Values followed by an asterisk are significantly different from the control $(P<0.05)$ by paired $t$-test analysis.

\begin{tabular}{|lcc|}
\hline \multirow{2}{*}{$\begin{array}{l}\text { Days } \\
\text { of exposure }\end{array}$} & \multicolumn{2}{c|}{ Feeding punctures } \\
\cline { 2 - 3 } & Control diet & $\mathbf{1 0 0 0}$ p.p.m. restrictocin \\
\hline 2 & $8.9 \pm 1.5$ & $2 \cdot 6 \pm 0 \cdot 7^{*}$ \\
4 & $23.5 \pm 3 \cdot 9$ & $8 \cdot 2 \pm 2 \cdot 0^{*}$ \\
\hline
\end{tabular}

reduced approximately threefold compared to the control diet (Table 4).

\section{Digestion assays}

Digestion of restrictocin by $H$. zea midgut homogenates could be discerned on native polyacrylamide gels at a homogenate dilution of 1:10, while digestion by the Spo. frugiperda midgut homogenate was only discernible with undiluted homogenate. H. zea and Spo. frugiperda appeared to digest the restrictocin differently. An obvious secondary band was noted with the $H$. zea homogenate, while no secondary bands were noted with the Spo. frugiperda homogenates. In these latter digestions, a smear of partially degraded protein was observed instead.

\section{DISCUSSION}

C. freemani adults fed upon colonies of $A$. nidulans and $A$. restrictus. The phialides of the conidiophores and the emerging, immature conidia of $A$. restrictus were not eaten during a particular time frame. A dramatic buildup of restrictocin is seen upon the surfaces of these structures during this same time frame (Brandhorst \& Kenealy,
1992). This suggests an involvement of the restrictocin in the deterrence of insect feeding. Fungi are an attractive food source for insects, particularly the fruiting bodies, which are rich in nitrogen and energy (Southwood, 1973), so it would be an advantage for the fungus to develop mechanisms to protect these structures.

Adult C. freemani were not killed by restrictocin at 1000 p.p.m., but they exhibited an aversion to restrictocin in their diet, as did Sit. zeamais. Larvae of $C$. freemani and Spo. frugiperda, but not $H$. zea, were killed by the toxin. The ability of gut proteinases to hydrolyse ricin and saporin appeared to explain the lack of susceptibility of caterpillar larvae to these toxins (Gatehouse et al., 1990). The difference in effect of restrictocin on Spo. frugiperda vs $H$. zea may be at least partly explained by different rates of digestion. At equivalent protein concentrations under the conditions used, the $H$. zea midgut homogenates were able to digest restrictocin approximately 10 times faster than those of Spo. frugiperda.

Restrictocin may act in concert with one or several of the other compounds present in the fungi to effectively deter feeding by adult $C$. freemani. The results of our experiments with pure restrictocin indicated that restrictocin by itself, when present at concentrations of 1000 p.p.m., deterred feeding by adult $C$. freemani. The natural concentration of restrictocin coating fungal phialides may be in excess of 1000 p.p.m., and was estimated at 1400 p.p.m. by calculating the volume and number of phialides per plate at maximum restrictocin production (Brandhorst \& Kenealy, 1992).

Restrictocin may protect $A$. restrictus conidiophores from insect feeding by one or more mechanisms. Accumulation of a lethal or sublethal dose of the toxin by fungivorous insects could check extensive feeding upon toxin-coated conidiophores. Alternatively, restrictocin toxicity may be targeted to specific cells. Poisoning cells of the gut lining might cause sufficient gastric distress to inhibit further feeding, while a specificity to taste receptor cells could hamper their normal functioning sufficiently to produce 
an antifeedant response. Finally, the restrictocin molecule could act as a insect repellent. While Bacillus thuringiensis crystal protein, for instance, acts as an anti-feedant for some insects (Gould et al., 1991), the lethal portions of the toxin have been found to be distinct from the portions that cause the anti-feedant/repellent response (Rajendran et al., 1994).

In tests of opaque- 2 and normal corn lines, the adults of Sitophilus oryzae were significantly more likely to be found with the opaque-2 lines (Gupta et al., 1970). The absence of the maize RIPs in the opaque-2 lines is now thought to be responsible for this increased susceptibility (Bass et al., 1992). It is possible that $S$. oryzae was able to sense the presence of the maize RIP, as we found for Sit. zeamais and restrictocin in the present study.

Once conidia have matured, restrictocin levels on the conidiophore heads diminish substantially (Brandhorst \& Kenealy, 1992) and the feeding deterrence effect wanes as well. It is probable that this is advantageous to the reproductive efficiency of the fungus, as fungivorous insects are an important vector for the dissemination of conidia. Investigators have noted that the majority of spores in the guts of Acaridae (Griffiths et al., 1959), Sitophilus granarius (Agrawal et al., 1957) and Carpopbilus spp. (Jewell, 1956; Moller \& Devay, 1968) remain undigested and pass through the gut maintaining full viability.

Prior tests with the plant-derived RIPs ricin and saporin indicated activity against beetle larvae, but not caterpillar larvae (Gatehouse et al., 1990). We also found that the Spo. frugiperda larvae were not affected after $48 \mathrm{~h}$ by the plantderived saporin and momordin. However, both beetle $(C$. freemani) larvae and some species of caterpillar larvae (Spo. frugiperda but not $H$. zea) were affected by restrictocin at 1000 p.p.m. The differing spectrum of insect toxicity of restrictocin compared to the plant-derived RIPs that have been tested suggests some fundamental differences in pharmacological activity. Fungal RIPs, such as restrictocin, may have ecologically relevant activity and value in insect control through genetic engineering that cannot be predicted based on comparisons with plant-derived RIPs.

\section{ACKNOWLEDGEMENTS}

This work was supported by NIGMS grant no. GM52706-01, J. Whittier Biologics and USDA-ARS.

Disclaimer: names are necessary to report factually on available data; however, the USDA neither guarantees nor warrants the standard of the product, and the use of the name by the USDA implies no approval of the product to the exclusion of others that may also be suitable.

\section{REFERENCES}

Agrawal, N. S., Christensen, C. M. \& Hodson, A. C. (1957). Grain storage fungi associated with the granary weevil. J Econ Entomol 50, 659-663.

Armitage, D. M. \& George, C. L. (1986). The effect of three species of mites upon fungal growth on wheat. Exp Appl Acarol 2, 111-124.
Bass, H. W., Webster, C, O'Brian, G. R., Roberts, J. K. M. (1992). A maize ribosome-inactivating protein is controlled by the transcriptional activator Opaque-2. Plant Cell 4, 225-234.

Brandhorst, T. \& Kenealy, W. R. (1992). Production and localization of restrictocin in Aspergillus restrictus. J Gen Microbiol 138, 1429-1435.

Brandhorst, T., Yang, R. \& Kenealy, W. R. (1994). Heterologous expression of the cytotoxin restrictocin in Aspergillus nidulans and Aspergillus niger. Protein Expr Purif 5, 486-497.

Conde, F. P., Fernandez-Puentes, C., Montero, M. T. V. \& Vazquez, D. (1978). Protein toxins that catalytically inactivate ribosomes from eukaryotic microorganisms. Studies on the mode of action of alpha sarcin, mitogillin and restrictocin: response to alpha-sarcin antibodies. FEMS Microbiol Lett 4, 349-355.

Cullen, D., Gray, G., Wilson, L., Hayenga, K., Lamsa, M., Rey, M., Norton, S. \& Berka, R. (1987a). Controlled expression and secretion of bovine chymosin in Aspergillus nidulans. Bio/technology 5, 369-376.

Cullen, D., Wilson, L. J., Grey, G. L., Henner, D. J., Turner, G. \& Ballance, D. J. (1987b). Sequence and centromere proximal location of a transformation enhancing fragment ans 1 from Aspergillus nidulans. Nucleic Acids Res 15, 9163-9175.

Dowd, P. F. (1988). Toxicological and biochemical interactions of the fungal metabolites fusaric acid and kojic acid with xenobiotics in Heliothis zea (F.) and Spodoptera frugiperda (J. E. Smith). Pestic Biocbem Pbysiol 32, 123-134.

Dowd, P. F. (1990). Responses of Carpopbilus bemipterus larvae and adults to selected secondary metabolites of maize. Entomol Exp Appl 54, 29-36.

Dowd, P. F. (1992a). Insect interactions with mycotoxin-producing fungi and their hosts. In Handbook of Applied Mycology, vol. 5, Mycotoxins in Ecological Systems, pp. 137-155. Edited by D. Bhatnagar, E. B. Lillehoj \& D. K. Arora. New York: Marcel Dekker.

Dowd, P. F. (1992b). Detoxification of mycotoxins by insects. In Molecular Mechanisms of Insecticide Resistance: Diversity Among Insects, pp. 264-275. Edited by C. A. Mullin \& J. G. Scott. Washington, DC: American Chemical Society.

Dowd, P. F. (1994). Enhanced maize (Zea mays L.) pericarp browning: associations with insect resistance and involvement of oxidizing enzymes. $J$ Chem Ecol 20, 2777-2803.

Dowd, P. F. (1995). Sap beetles and mycotoxins in maize. Food Addit Contam 12, 497-508.

Dowd, P. F. \& Weber, C. M. (1991). A labor-saving method for rearing a corn sap beetle, Carpophilus freemani Dobson (Coleoptera: Nitidulidae) on pinto bean-based diet. J Agric Entomol 8, 149-153.

Frey, D., Oldfield, R. J. \& Bridger, R. C. (1979). Color Atlas of Pathogenic Fungi. Chicago: Yearbook Medical Publishers.

Gatehouse, A. M. R., Barbieri, L., Stirpe, F. \& Croy, R. R. D. (1990). Effects of ribosome inactivating proteins on insect development differences between Lepidoptera and Coleoptera. Entomol Exp Appl 54, 43-51.

Gatehouse, A. M. R., Shi, Y., Powell, K. S., Brough, C., Hilder, V. A., Hamilton, W. D. O., Newell, C. A., Merryweather, A., Boulter, D. \& Gatehouse, J. A. (1993). Approaches to insect resistance using transgenic plants. Philos Trans R Soc Lond B 342, 279-286.

Gould, F., Anderson, A., Landis, D. \& Van Mellaert, H. (1991). Feeding behavior and growth of Heliothis virescens larvae on diets containing Bacillus thuringiensis formulations or endotoxins. Entomol Exp Appl 58, 199-210.

Griffiths, D. A., Hodson, A. C. \& Christensen, C. M. (1959). Grain storage fungi associated with mites. $J$ Econ Entomol 52, 514-518. 
Gupta, S. C., Asnani, V. L. \& Khare, B. P. (1970). Effect of the opaque-2 gene in maize (Zea mays L.) on the extent of infestation by Sitophilus oryzae L. J Stored Prod Res 6, 191-194.

Jewell, F. F. (1956). Insect transmission of oak wilt. Pbytopatbology 46, 244-257.

Jimenez, A. \& Vazquez, D. (1985). Plant and fungal protein and glycoprotein toxins inhibiting eukaryote protein synthesis. Annu Rev Microbiol 39, 649-672.

Moller, W. J. \& Devay, J. E. (1968). Insect transmission of Ceratocysus fimbriata in deciduous fruit orchards. Pbytopathology 58, 1499-1508.

Rajendran, N., Sivamani, E. \& Jayaraman, K. (1994). Expression of the insecticidal crystal protein gene from a Gram-positive Bacillus thuringiensis in a Gram-negative Pseudomonas fluorescens mediated by protoplast fusion. FEMS Microbiol Lett 122, 103-108.

Southwood, T. R. E. (1973). The insect/plant relationship-an evolutionary perspective. In Insect/Plant Relationships, pp. 3-29. Edited by H. F. van Emden. New York: Wiley.
Stirpe, F. \& Barbieri, L. (1986). Ribosome-inactivating proteins up to date. FEBS Lett 195, 1-8.

Tate, K. G. \& Ogawa, J. M. (1975). Nitidulid beetles as vectors of Monilia fructicola in California stone fruits. Pbytopathology 65, 977-983.

Wicklow, D. T. (1988). Metabolites in the coevolution of fungal chemical defence systems. In Coevolution of Fungi with Plants and Animals, pp. 173-201. Edited by K. A. Pirozynski \& D. L. Hawksworth. New York: Academic Press.

Wicklow, D. T., Dowd, P. F., Tepaske, M. R. \& Gloer, J. B. (1988). Sclerotial metabolites of Aspergillus flavus toxic to a detritivorous maize insect. Trans Br Mycol Soc 91, 433-438.

Wright, V. F., Vesonder, R. F. \& Ciegler, A. (1982). Mycotoxins and other fungal metabolites as insecticides. In Microbial and Viral Pesticides, pp. 559-583. Edited by E. Kustak. New York: Marcel Dekker.

Received 20 December 1995; accepted 11 January 1996. 